\title{
Smart Monitoring of the Status of Driver Using the Dashboard Vehicle Camera
}

\author{
Dr. S. Praveen Chakkravarthy \\ Associate professor-ECE, CVR College of Engineering, Hyderabad, India \\ too.spc@gmail.com
}

\begin{tabular}{|c|c|}
\hline Article History & Abstract \\
\hline $\begin{array}{l}\text { Article Submission } \\
30 \text { November } 2019 \\
\text { Revised Submission } \\
25 \text { Feb } 2020 \\
\text { Article Accepted } \\
12 \text { March } 2020 \\
\text { Article Published } \\
31^{\text {st }} \text { March } 2020\end{array}$ & $\begin{array}{l}\text { One of the potential approaches to lessen the quantity of mishaps in rush hour } \\
\text { gridlock is to follow the driver's condition. In this paper, a new driver observing } \\
\text { calculation, which works on a video caught from the camera situated in the } \\
\text { instrument board in the vehicle, is introduced. The calculation analyzes the nearness } \\
\text { of the driver's head in each edge and checks whether the driver has bizarrely long } \\
\text { closed eyes, regardless of whether the driver looks sideways, and whether the } \\
\text { driver's head has dropped for quite a while. The calculation is tried utilizing ten } \\
\text { unique recordings recorded under various light conditions. The calculation for the } \\
\text { most part accomplishes superior, while for a few lighting conditions a specific } \\
\text { calculation redesigning would be vital to keep up an elevated level of precision. } \\
\text { Keywords: Rush Hour, Grid Lock, Smart Monitoring, Camera }\end{array}$ \\
\hline
\end{tabular}

\section{Introduction}

Every year the amount of deaths and injuries are increasing drastically by road accidents. Mostly they are due to human errors like drinking \& driving, over speeding, drowsiness etc. People can avoid the accidents caused by drinking alcohol. Unfortunately, it is not easy to avoid the accidents caused by drowsiness, as it is uncertain. People who drive long distance tend to fall asleep during less traffic hours or when travelling on straight roads. The National Sleep Foundation declares Drowsy Driving Prevention Week is November 3-10. Though government is spreading awareness it is difficult to avoid this. Hence a system like this is needed to monitor the driver's state continuously. Care-max for drowsiness detection and notification to address this issue. The proposed system involves a camera which continuously monitors the eyes of the driver. There are already existing solutions for this challenge. But they result in more computational complexity and are costly too. The algorithm they use is also not very efficient and is not accurate. But our proposed system uses two vertical eye landmarks to find the aspect ratio. This is done using computer vision. The level of drowsiness is determined and alerts the driver by the alarm sound. This promises accuracy and cost efficiency. This will be very helpful in reducing the amount of accidents caused by driver falling asleep [1].

In addition to drowsiness detection and alerting, this system comes as a package that includes a mobile application. This mobile app has some settings that user enters for the first time only. The settings include interval of wheel air checking, wheel alignment, radiator check and service date of the car. This app sends the configured information to the system [2].

The system uses a voice assistance module that remind user when to check air or when to put car under next service. This app communicates to the system using IOT. Hence, our system "CARE-MAX for drowsiness detection and car health notification" promises to give better results [3].

All the objectives are shown below:

a. To design and develop a system that detects drowsiness and alerts the driver, controlled by Raspberry pi.

b. To design a mobile application for vehicle maintenance using android studio.

c. To implement the drowsiness detection algorithm and successfully calculate the eye aspect ratio.

d. To develop the prototype of this system. 
Several of method exist for drowsiness detection and check the drowsiness state while driving and alerts the driver if they are drowsy. The system uses variety of sensors to detect the state. They are not accurate and inefficient. The cost of the system is unaffordable.

\section{Existing techniques}

In [1], Detecting drowsiness of the driver needs a sensor to capture the face of the driver. In case the captured face doesn't fit in the frame, an alert is sent to the person in charge and the driver to adjust accordingly. The GPS/GPRS module is given as an input to the controller. The whereabouts of the driver is sent to the medical assistance. Speed of the moving car is measured using accelerometers. This information is preserved in the application. The camera which is the video sensor captures the face of the person driving the car and by calculating the blinking frequency of the person, authors conclude if the person is drowsy. If the frequency of blinking is more, the person is assumed to be drowsy [4].

In order to find the drowsiness, [5], the eye blink sensor is employed. Drowsiness is detected using a glass similar to a reading glass with eye blink sensor that buzzers in case the person driving the car is drowsy. They used the sensor like tilt sensor, eye blink sensor to detect and process. In [3], they illustrated that there are several methods to find if the car driver is sleepy. Various parameters can be used for the detection. It can be behavioural changes of the person driving or psychology of movements of the person. Here it is based on behavioural parameters of the person driving. Those parameters are tilt of head, blinking of eyes, closure of eyes, yawning. Author uses image processing to provide a solution for finding if driver is sleepy at the soonest and gets time to avoid the danger [7][8]. In [6], they address the issue of road accidents caused by drowsiness of driver. The level of drowsiness in drivers is calculated. They utilise pi cam and a raspberry pi series 3 . Author utilises the tilt rate of head and blink rate of the eyes to find if the person driving is sleepy. The region of interest is used to detect the face and estimate fatigue. The gaze detection is used to detect the drowsy state. The work presented in [5], is based on the list given by the Association for Safe International Road Travel about statistics of road accidents [9]. A non-profit and non-government organisation that improves the personal safety of travellers on the roads. They also listed the safety tools [10].

\section{Proposed Methodology}

The driver fatigue or tiredness detection system also called as Rest Assist. This system is already available in new Volkswagen and Skoda. This extracts the lowering focus rates by measuring the clutch and steering wheel usage. This paper [7], describes the facts and stats regarding the sleepy driving. These were provided by National Highway Traffic Safety Administration. It is illustrated that when the people are driving drowsy, 42 percentage of people polled that they become high on stress, 32 percentage ends up impatient and 12 percentage drive faster. These problems can be overcome by care-max drowsiness detection and assist them with a voice command.

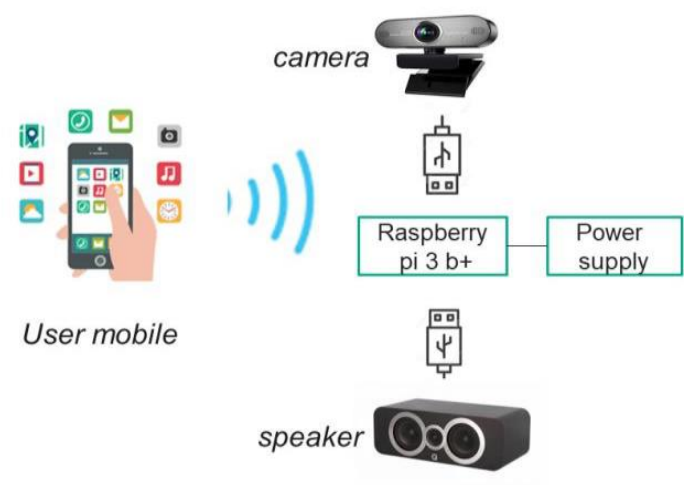

Fig 1: Block diagram of care max

In this area the new proposed calculation for driver observing, in view of facial investigation is depicted. Our drowsiness detection model reduces the accident counts by the driver due to sleepy state. The group of hardware 
components is used for the detection of drowsy and android application is used for the notification which involves interval of wheel air checking, wheel alignment, radiator check and service date of the car.

To observe the face of the driver, a live video captured through web camera is provided as an input to the controller named raspberry pi $3 b+$. The stream of face in video is monitored and processed by the raspberry pi $3 b+$. The landmarks detection on face using Dlib and openCV is employed. Dlib 5-points facial landmark detector is a technique that reduces the 68-point detector identifies areas which includes eyes, eyebrows, nose, mouth and jawline to 2 points for the left eye, 2 points for the right eye and 1 points for the nose. It also increases the speed of detection up to 8-10\% than the original version. But to build face detection for the drowsiness detection we use 68-point detector to get more points on eyes where only two points per eye in 5-point detector. It increases the accuracy of the detection. The face detection model: i. Haar cascades: It is faster but has low accuracy. ii. HOG + Linear SVM detector: It is much precise to the Haar cascades with lower false positives and also requires minimum arguments. The Facial landmark detector is loaded and live stream is initialized. Each frame taken from the live video that streamed is processed then converted to grayscale image. We use haar cascades model to detect the faces in grayscale image. Each face is detected and visualized.

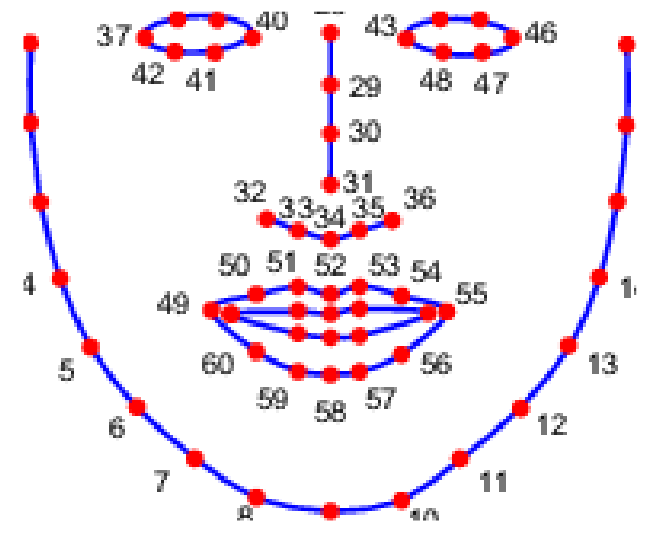

Fig 2: Facial landmarks

The driver's face is captured. The drowsiness level of drowsiness of the driver is predicted by the eye blink rate. The eye landmarks should be extracted to calculate blink rate. The landmarks of both the eyes are extracted by using facial landmarks indexes. The extraction points of the left eye and the right eye is achieved easily. 4.3 EAR PROCESSING The face is detected and the eyes are extracted from the image. To find the eye blink rate and to predict the drowsiness eye aspect ratio (EAR) is calculated. Eye aspect ratio is given by the distances between the vertical landmarks of the eye divided by the distance between the horizontal landmarks of the eye.

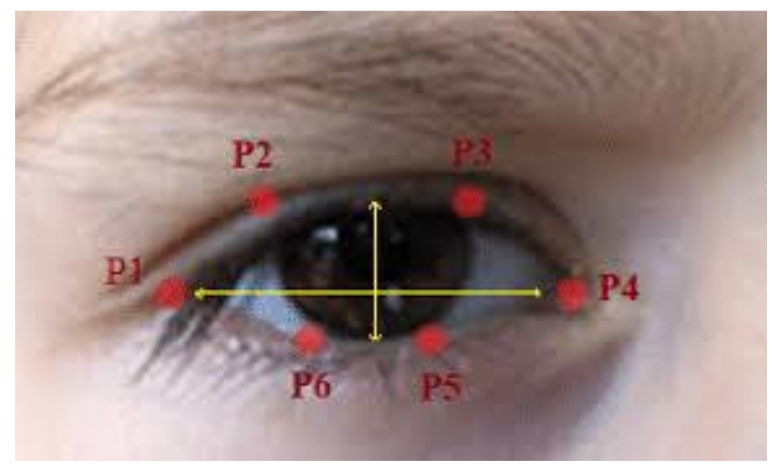

Fig 3: Eye Aspect Ratio

The Euclidean distances among the two sets of vertical eye landmarks are calculated. The Euclidean distances among the horizontal landmarks of the eyes are calculated. Then the (EAR) eye aspect ratio is computed. 


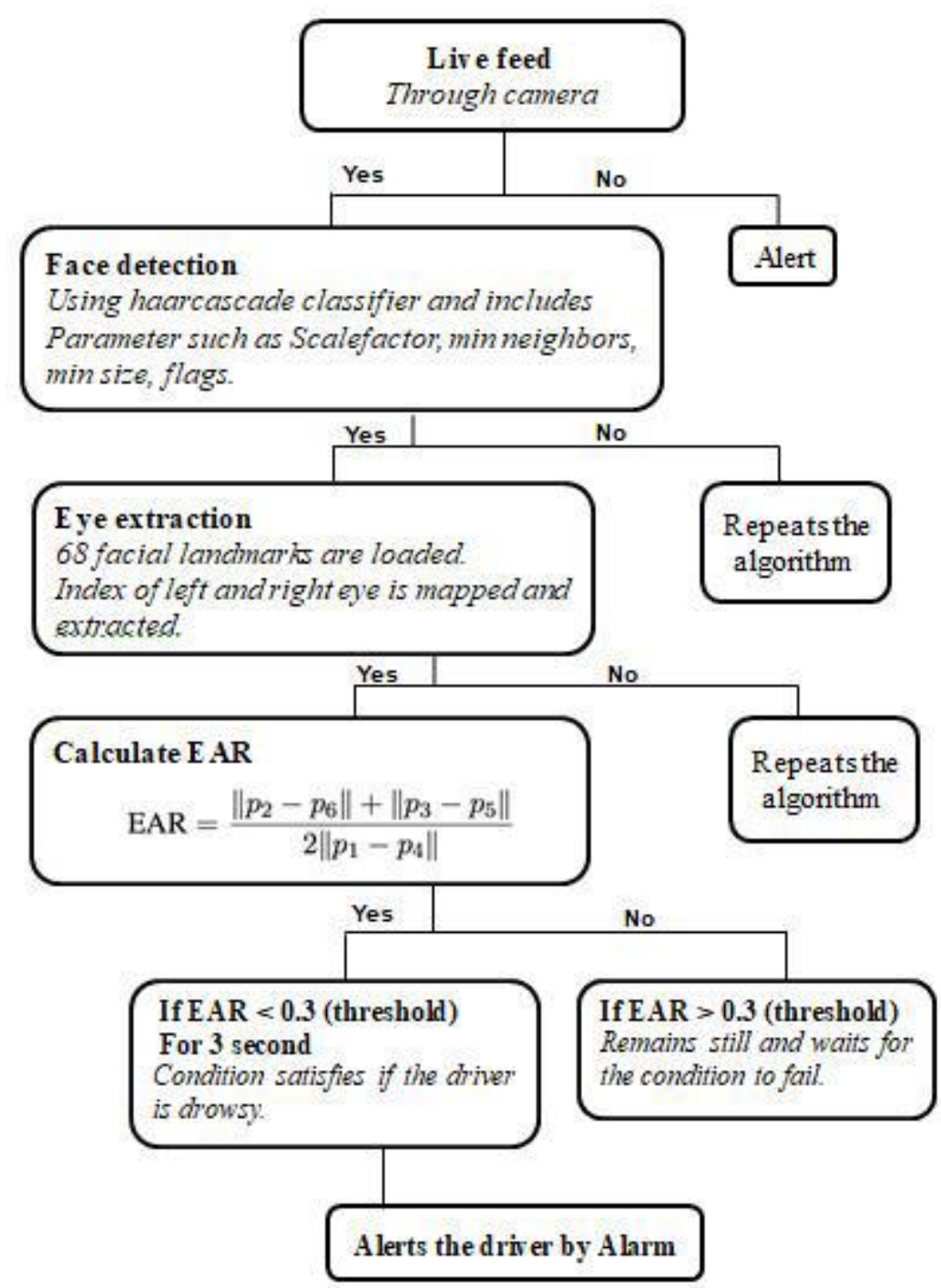

Fig 4. Flow chart of system

If the state of eye is not closed, the measure of (EAR) eye aspect ratio 13 will be constant. For every blink, the measure of (EAR) eye aspect ratio will be a fall rapidly towards zero. If the state of eye is close, the measure of (EAR) eye aspect ratio will be constant but smaller than when it is open.

$$
\mathrm{EAR}=\frac{\left\|p_{2}-p_{6}\right\|+\left\|p_{3}-p_{5}\right\|}{2\left\|p_{1}-p_{4}\right\|}
$$

The camera along with controller is placed in front of the person driving. So that the face of the person driving is clear and they are no disturbance in driving. The driver sign into the application and configured all the setting. Through the live video the face is detected and eye is extracted. Considering the value of eye aspect ratio (EAR) the drowsiness of the driver is detected and alarms with a sound if the value of EAR is decreased. The controller connects with application through IoT. The remembrance of the details such as services and maintenance will be intimate via voice command.

\section{Simulation Results}

This section provides the experimentation results of the drowsiness detection. Getting the highest accuracy is the challenge due to the poor lighting and unclear frame. The camera detects the face of the driver and continuously 
monitors the state of eye. The eye aspect ratio is measured for each frame which is used to find the state of the driver is detected.
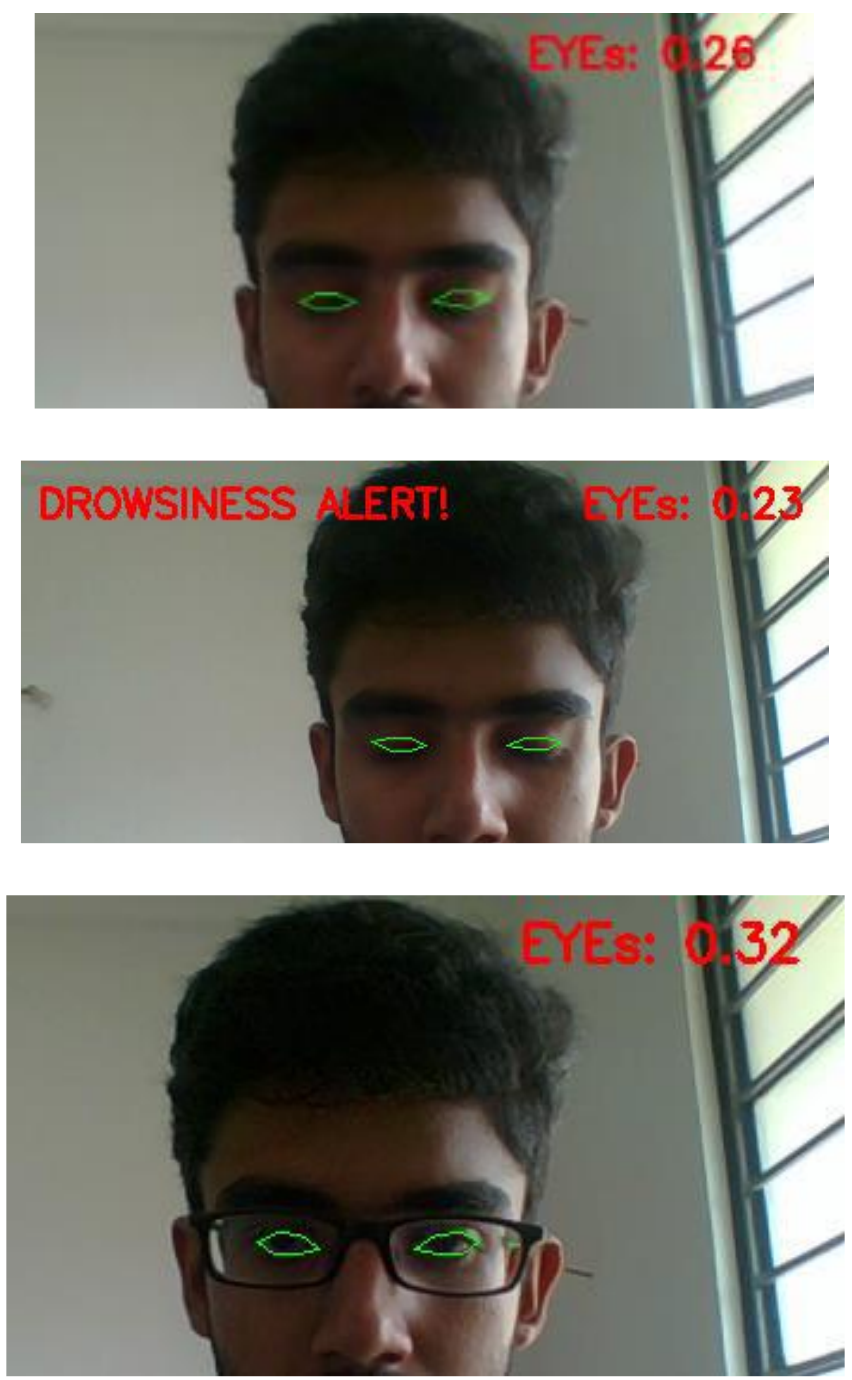

Fig 5: Eye extraction, Drowsiness detection and Eye extraction from the frame

The eye blink rate is normal and the person is in normal state. The eye is extracted and eye aspect ratio is measured. Since the eye aspect ratio is constant and high, the system doesn't give the drowsiness alert with the alarm sound.The eye blink rate is abnormal and the person is in drowsy state. The eye is extracted and eye aspect ratio is calculated. The eye ratio is reduces largely and stays constant. If it remains for some seconds, the system gives the drowsiness alert with the alarm sound. The eye is extracted from the frame though he wears specs. The drowsiness of the person is detected though the person wears specs.

This section concentrates on the observation of success rate in the normal condition. Normal condition Number of attempts Success counts Percentage Eye blink 201575 Table 1. Detection percentage in normal conditions. This section concentrates on the observation of success rate in the normal condition.

Table 1. Detection percentage in normal conditions

\begin{tabular}{|c|c|c|c|}
\hline Normal condition & Number of attempts & Success counts & Percentage \\
\hline eye blink & 20 & 15 & 75 \\
\hline
\end{tabular}


This section focuses on the observation of success rate with some special condition such as person wearing glasses and hair covering the person.

Table 2. Detection percentage in other parameters

\begin{tabular}{|c|c|c|c|}
\hline Parameter & Number of attempts & Success counts & Percentage \\
\hline Person wearing glasses & 20 & 7 & 35 \\
\hline Hair covering the person's face & 20 & 5 & 25 \\
\hline
\end{tabular}

\section{Detection process algorithm is shown below}

Initiate the process

Define the parameters

$$
\begin{array}{ll}
\text { 1. } & \text { TOA } \\
\text { 2. } & \text { TDOA } \\
\text { 3. } & \text { CSI }
\end{array}
$$

State_to_location: PPCC dict (PPC, ASR)

for the available data rate

Preprocessing: Standardization

Discount factor

Learning rate

Find optimal power:

Define Discount factor (a) and learning rate (b)

Initialize\#

$a=0.75$

$b=0.9$

Now:

Define helper function $h(a)$

\section{While optimal carrier:}

\#Define 2 argument

\#Start from $1^{\text {st }}$ control bit

\#End in (n-1)st Data bit

$>\quad \boldsymbol{h}(\boldsymbol{a})$ should return the optimal power in the burst as a function of PPCC NEW STATE

Preprocessing: Optimization

$Q$ value initialization

Reward matrix initialization

NEW STATE initialization

Start:

$$
\text { Optimize ASR }
$$

Initializing $Q$ values to Zero

$Q=h p$ array $(h p . Z e r o(1, n-1))$

Extend reward matrix entity

reward_value $=$ hp.copy $($ rewards $)$.

Hp-Starting \&Ending location.

Training $(h p, A S R)$

$\boldsymbol{h} \boldsymbol{h}$ should return the TDOA (Time delay of arrival) in an interference limited system for $(1, n-1)$

location.

\section{Priority:}

Starting integer:0

Larger integer ending location: 999

The above integer goes through 0 to $b$ with a to return the ASR the ending state being the highest one. NEW STATE 


\author{
Now: for $D$ in range 0 to 999: \\ Pick the states random $(0,999)$ \\ Current state $=$ hp. random. rand int $(0,999)$
}

\title{
V. Conclusion
}

This paper focuses on the drowsiness detection and notifies the driver regarding the wheel air checking, wheel alignment, radiator check and service date of the car. The system gets the live video as the input and detects the facial landmarks in the each frame. The eye is extracted and monitored. Depends on the eye aspect ratio state of the person is detected. If the person is in drowsy state system alerts by the alarm sound. Based on the experimental results, the volume of the model used to detect the face and eye is small. Hence, it can be integrated into car for the advanced automation. The future work will be focusing on the estimation of human behaviour such as yawning and distraction of the driver. Also the system can be integrated with the braking system to slower the speed of the vehicle when the drowsiness can be detected.

\section{References}

[1] Human Drowsiness Detection system, International Journal of Engineering and Advanced Technology (IJEAT), ISSN: 2249 - 8958, Volume-8 Issue-4, April, 2019

[2] Driver Drowsiness Detection System for Vehicle Safety International Journal of Innovative Technology and Exploring Engineering, ISSN: 2278-3075, Volume-8, Issue- 6S4, April 2019

[3] Real Time Driver Drowsiness Detection Based on Driver's Face Image Behavior Using a System of Human Computer Interaction Implemented in a Smartphone, January 2018

[4] Driver Drowsiness Detection using Eye-Closeness Detection, Oraan Khunpisuth, Taweechai Chotchinasri, Varakorn Koschakosai and Narit Hnoohom. 2016 12th International Conference on SignalImage Technology \& Internet-Based Systems.

[5] Association for Safe International Road Travel (ASIRT), Road Crash Statistics, 2016.

[6] Don't sleep and drive - VW's fatigue detection technology, Jan, T, Karnahl, Seifert,

[7] K. , Hilgenstock, J. , \& Zobel, R, 2016. Drowsy Driving, Facts and Stats: Drowsy Driving - Stay Alert, Arrive Alive, 2016.

[8] S. M. Sarala, D. H. Sharath Yadav and A. Ansari, "Emotionally Adaptive Driver Voice Alert System for Advanced Driver Assistance System (ADAS) Applications," 2018 International Conference on Smart Systems and Inventive Technology (ICSSIT), Tirunelveli, India, 2018, pp. 509-512, doi: 10.1109/ICSSIT.2018.8748541.

[9] H. K. Dua, S. Goel and V. Sharma, "Drowsiness Detection and Alert System," 2018 International Conference on Advances in Computing, Communication Control and Networking (ICACCCN), Greater Noida (UP), India, 2018, pp. 621-624, doi: 10.1109/ICACCCN.2018.8748448.

[10] C. G. Babu, R. H. Kumar, P. Sampath, P. Bhuvaneshwari and M. Dharanya, "Analysis of Conscious Alert System from EEG data using LabView," 2018 International Conference on Computer Communication and Informatics (ICCCI), Coimbatore, 2018, pp. 1-3, doi: 10.1109/ICCCI.2018.8441260. 